\title{
Bifurcation to square-wave switching in orthogonally delay-coupled semiconductor lasers: Theory and experiment
}

\author{
C. Masoller, ${ }^{1}$ D. Sukow, ${ }^{2}$ A. Gavrielides,${ }^{3}$ and M. Sciamanna ${ }^{4}$ \\ ${ }^{1}$ Departament de Fisica i Enginyeria Nuclear, Universitat Politecnica de Catalunya, Colom 11, ES-08222 Terrassa, Barcelona, Spain \\ ${ }^{2}$ Institute for Cross-Disciplinary Physics and Complex Systems, Campus Universitat de les Illes Balears, ES-07122 Palma de Mallorca, Spain \\ ${ }^{3}$ Air Force Research Laboratory, AFRL/EOARD, 86 Blenheim Crescent, Ruislip Middlesex HA4 7HB, United Kingdom \\ ${ }^{4}$ Optics and Electronics (OPTEL) Research Group, Laboratoire Matériaux Optiques, Photonique et Systemes (LMOPS), Supélec, \\ 2 Rue Edouard Belin, FR-57070 Metz, France
}

(Received 8 April 2011; published 22 August 2011)

\begin{abstract}
We analyze the dynamics of two semiconductor lasers with so-called orthogonal time-delayed mutual coupling: the dominant TE $(x)$ modes of each laser are rotated by $90^{\circ}$ (therefore, TM polarization or $y$ ) before being coupled to the other laser. Although this laser system allows for steady-state emission in either one or in both polarization modes, it may also exhibit stable time-periodic dynamics including square waveforms. A theoretical mapping of the switching dynamics unveils the region in parameter space where one expects to observe long-term time-periodic mode switching. Detailed numerical simulations illustrate the role played by the coupling strength, the mode frequency detuning, or the mode gain to loss difference. We complement our theoretical study with several experiments and measurements. We present time series and intensity spectra associated with the characteristics of the square waves and other waveforms observed as a function of the strength of the delay coupling. The experimental observations are in very good agreement with the analysis and the numerical results.
\end{abstract}

DOI: 10.1103/PhysRevA.84.023838

PACS number(s): 42.55.Px, 42.60.Mi, 42.65.Pc

\section{INTRODUCTION}

In many different configurations, the dynamics of a semiconductor laser is significantly perturbed by a time-delayed optical coupling or optical feedback. The naturally damped oscillatory transient dynamics (so-called relaxation oscillations) may become undamped and, depending on the coupling strength and time-delay values, the laser diode can exhibit a rich variety of bifurcation scenarios possibly leading to optical chaos [1-3]. An in-depth understanding of these instabilities leading to laser nonlinear dynamics is crucial in several applications that require a stable laser output, or by contrast that target the development of a controllable periodic [4] or chaotic light source [5]. Moreover, the conclusions drawn from the laser system analysis can typically be extended to other systems in biology, chemistry, mechanics, and economy, where the combination of nonlinearity and time delay is ubiquitous [6].

Even more richness in the laser dynamics can be observed in the presence of mode competition and noise, in addition to time delay. In edge-emitting lasers (EELs), time-delayed optical feedback or coupling can enhance lasing in the many longitudinal modes and lead to either in-phase or antiphase (anticorrelated) modal intensity dynamics [7-12]. In vertical-cavity surface-emitting lasers (VCSELs), timedelayed optical feedback or optical coupling can modify the intrinsic competition between orthogonal polarization modes and induce or suppress polarization switching [13-18]. The mode competition dynamics in the presence of time delay are moreover very sensitive to noise (spontaneous emission noise or external noise source), which not only may drive transitions between stable states but also may lead to constructive dynamical effects such as coherence resonance [19-22] and stochastic resonance [23-25].
We focus here on such a particular example of a twostate laser system in the presence of time-delayed coupling and noise. It comes from a recent experiment [26] where two Fabry-Perot multi-quantum-well EELs lasing with TE polarizations are mutually coupled through TM polarizations. More specifically, the dominant TE mode of each laser is rotated by $90^{\circ}$ before being delayed coupled to the other laser. Such a configuration (hereby called orthogonal mutual coupling) allows for the observation of square-wave switching between TE and TM modes simultaneously in the two lasers, at a period related to the coupling time delay and with duty cycles that are tunable as a function of the coupling strength and pump currents. The possibility to generate all optically a stable and tunable pulsating laser output has attracted a lot of attention in recent years. Similar examples of sinusoidal to square-wave high-frequency pulsating dynamics have been observed in VCSELs with so-called polarization self-modulation [27-31], in EELs with polarization-rotated optical feedback $[10,12,32-35]$, and in optoelectronic feedback systems [36].

In this paper, we first make a detailed theoretical study of the square-wave dynamics in EELs with orthogonal mutual coupling, as observed experimentally in Ref. [26]. As the coupling strength increases, the laser dynamics exhibits either a steady two-mode dynamics (mixed-mode solution) or a steady one-mode dynamics (pure-mode solution), but both fixed points bifurcate to pulsating dynamics that evolve into either transient or long-term stable square-wave dynamics. The square-wave dynamics is characterized by their transient time and averaged intensities in TE and TM polarizations (later called $x$ and $y$ polarization modes). These quantities are then mapped as a function of the cavity-loss difference between $x$ and $y$ modes, of the coupling strength, and of the $x-y$ polarization frequency detuning. These mappings then allow 
us to emphasize those regions of the parameter space where one can expect to observe long-term stable square-wave dynamics. By showing different complex dynamics and by analyzing the influence of several important laser parameters, our numerical study complements the earlier experimental observations of stable square-wave switching and therefore motivates further measurements. In a second part of this paper, we present several measurements of time-series and rf spectra of the laser intensities in the coupled system. They not only illustrate the symmetric and nonsymmetric switching solutions found in numerical simulations, but also confirm the bifurcation scenario leading to square-wave switching and the coexistence of several solutions (multistability), as found in the theoretical bifurcation analysis.

Our paper is organized as follows. Section II details the theoretical model and presents the steady-state solutions. Section III discusses the numerical results, obtained through direct numerical integration of the rate equations. Section IV shows the experimental results of stable symmetric and nonsymmetric square-wave switching, and regular or complex self-pulsations. Finally, Sec. V summarizes our main conclusions.

\section{MODEL}

\section{A. Model equations}

The rate equations describing two identical semiconductor lasers mutually coupled through polarization-rotated optical injection are

$$
\begin{aligned}
\frac{d E_{x, i}}{d t}= & k(1+j \alpha)\left(g_{x, i}-1\right) E_{x, i}+\sqrt{\beta_{s p}} \xi_{x, i}, \\
\frac{d E_{y, i}}{d t}= & j \delta E_{y, i}+k(1+j \alpha)\left(g_{y, i}-1-\beta\right) E_{y, i} \\
& +\eta E_{x, 3-i}(t-\tau) e^{-j \omega_{0} \tau}+\sqrt{\beta_{s p}} \xi_{y, i}, \\
\frac{d N_{i}}{d t}= & \gamma_{N}\left[\mu-N_{i}-g_{x, i} I_{x, i}-g_{y, i} I_{y, i}\right] .
\end{aligned}
$$

Here, $i=1$ and $i=2$ denote the two lasers, $E_{x}$ and $E_{y}$ are orthogonal linearly polarized slowly varying complex amplitudes (corresponding, respectively, to TE and TM polarizations), and $N$ is the carrier density. In the absence of optical coupling, the emission frequency of the two lasers is the same $\left(\omega_{0}\right)$, which is the frequency of the $x$ polarization and is taken as reference frequency. $\delta$ is the frequency detuning between the $x$ and $y$ polarizations. The modal gains include self- and cross-saturation coefficients:

$$
\begin{aligned}
& g_{x, i}=N_{i} /\left(1+\epsilon_{x x} I_{x, i}+\epsilon_{x y} I_{y, i}\right), \\
& g_{y, i}=N_{i} /\left(1+\epsilon_{y x} I_{x, i}+\epsilon_{y y} I_{y, i}\right) .
\end{aligned}
$$

Other parameters are as follows: $k$ is the field decay rate, $\gamma_{N}$ is the carrier decay rate, $\alpha$ is the linewidth enhancement factor, $\beta$ is the linear loss anisotropy, $\beta_{s p}$ is the noise strength, $\xi_{x, y}$ are uncorrelated Gaussian white noises, and $\mu$ is the injection current parameter, normalized such that the solitary threshold is at $\mu_{t h}=1$. The parameters of the polarization-orthogonal mutual coupling are $\eta$ and $\tau$, which represent the coupling strength and the delay time, respectively. In Eq. (1), the phase factor $e^{-j \omega_{0} \tau}$ corresponds to a phase accumulated due to the propagation from laser 1 to laser 2 and vice versa and can be eliminated by a redefinition of $E_{x, 1}$ and $E_{x, 2}$. Therefore, it does not affect the dynamics and will be ignored.

\section{B. Steady-state solutions}

The model has two types of steady states:

(a) Mixed-mode solution, in which both lasers emit in $x$ and $y$ polarization simultaneously. The mixed solution is invariant under the exchange of lasers 1 and 2, i.e., $I_{1 x}=I_{2 x}, I_{1 y}=I_{2 y}$, and $N_{1}=N_{2}$.

(b) Pure-mode solution, in which one laser emits only in the $x$ polarization, while the other only on the orthogonal polarization. The pure-mode solutions are also symmetric with respect to an exchange of lasers. In essence, the laser that emits in the $x$ polarization becomes the master laser which drives the other laser into the orthogonal polarization through the rotated optical injection.

These steady states can be computed as follows. (a) In the symmetric mixed states, we have that $I_{1 x}=I_{2 x}=I_{x}, I_{1 y}=$ $I_{2 y}=I_{y}$, and $N_{1}=N_{2}=N$. The intensities $I_{x}$ and $I_{y}$ can be calculated from

$$
\mu-1-\left(1+\epsilon_{x x}\right) I_{x}-\epsilon_{x y} I_{y}-g_{y} I_{y}=0
$$

and

$$
\eta^{2}=\frac{I_{y}}{I_{x}}\left\{\left[\delta+k \alpha\left(g_{y}-1-\beta\right)\right]^{2}+k^{2}\left[g_{y}-1-\beta\right]^{2}\right\},
$$

where

$$
g_{y}=\frac{1+\epsilon_{x x} I_{x}+\epsilon_{x y} I_{y}}{1+\epsilon_{y x} I_{x}+\epsilon_{y y} I_{y}} .
$$

Equation (7), which is obtained from the carrier density equation eliminating $N$ by taking into account that

$$
g_{x}=\frac{N}{1+\epsilon_{x x} I_{x}+\epsilon_{x y} I_{y}}=1,
$$

can be solved for $I_{x}$ with $I_{y}$ as a parameter, $0<I_{y}<\mu-1$. Then, evaluating Eq. (8) (that results from solving the field equations taking into account that the frequency of the $y$ polarization locks to the frequency of the $x$ polarization), we obtain the coupling strength $\eta$ as a function of $I_{x}$ and $I_{y}$. With $I_{x}$ and $I_{y}$, the carrier density can be calculated from Eq. (10).

The mixed states become unstable due to a Hopf bifurcation. Numerically, we find that the symmetry property $I_{1 x}=$ $I_{2 x}, I_{1 y}=I_{2 y}$, and $N_{1}=N_{2}$ persists after the bifurcation, after which the intensities and carrier densities are time dependent.

(b) In the pure-mode solutions, the lasers emit orthogonal polarizations. We will refer to the laser that has $I_{x} \neq 0$ as "solitary" laser (the carrier density in this laser will be $N_{x}$ ), and we will refer to the laser that has $I_{y} \neq 0$ as "injected" laser (the carrier density in this laser will be $N_{y}$ ). For the solitary laser, the trivial steady state is

$$
\begin{gathered}
I_{x}=\frac{\mu-1}{1+\epsilon_{x x}}, \\
g_{x}=\frac{N_{x}}{1+\epsilon_{x x} I_{x}}=1 .
\end{gathered}
$$


For the injected laser, since the frequency of the $y$ polarization locks to the frequency of the injected $x$ polarization, $I_{y}$ and $N_{y}$ can be calculated from Eq. (8), where

$$
g_{y}=\frac{\mu}{1+I_{y}+\epsilon_{y y} I_{y}}=\frac{N_{y}}{1+\epsilon_{y y} I_{y}} .
$$

For $I_{y}>0$, we can evaluate Eqs. (8) and (13), and determine the coupling strength as a function of $I_{x}$ and $I_{y}$. With $I_{x}$ and $I_{y}$, we can calculate the carrier densities in the two lasers $N_{x}$ and $N_{y}$ from Eqs. (11) and (13).

\section{Analysis of the stability of the steady-state solutions}

Figure 1 displays the pure-mode (red online) and mixedmode (green online) stationary solutions for the $y$-mode intensity $I_{y}$ as a function of the coupling strength $\eta$. Together with the branches of stationary solutions, we plot the numerical solution, specifically, the value of $I_{y}$ at the extreme (maxima and minima) of $I_{x}$ as we increase (a) and then decrease (b) $\eta$ (the details of the simulations are presented in the next section). In Fig. 1(a), the coupling is gradually increased; in Fig. 1(b), the coupling is gradually decreased. The model parameters are such that, in the absence of coupling, the lasers emit only the $x$ polarization

Such a bifurcation diagram allows us to analyze the critical values of the coupling strength when the stationary solutions destabilize to time-periodic or more complicated intensity waveforms. The complete bifurcation scenarios will be discussed further in Sec. III.

In Fig. 1, there is only one branch of mixed-mode solution, as there is only one positive root when solving Eqs. (7) and (8) for $I_{y}$. For the pure-mode solutions, one or three positive roots are found when solving Eqs. (8) and (13) for $I_{y}$. Thus, the pure-mode branches correspond to an $S$ curve (in Fig. 1,
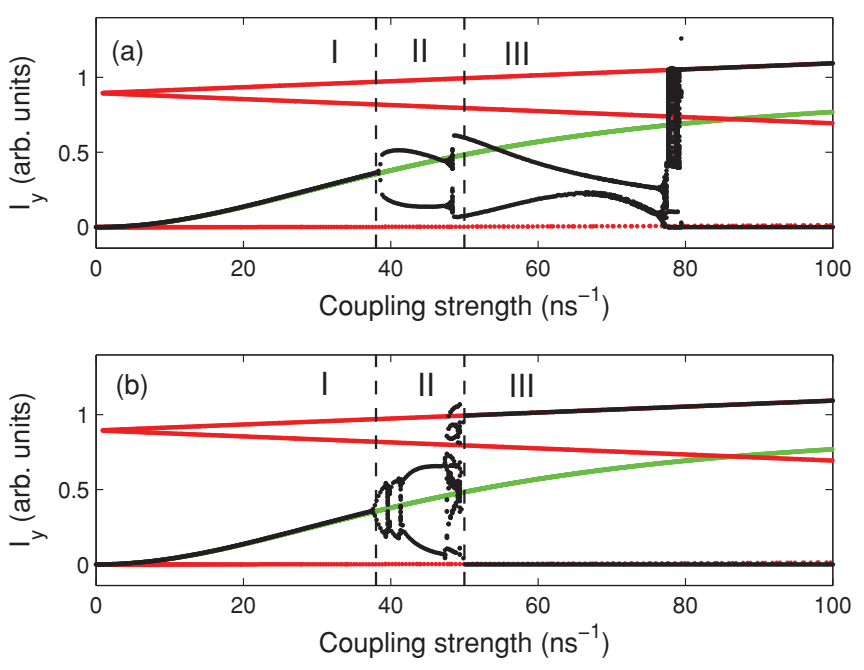

FIG. 1. (Color online) Intensity of the $I_{y}$ polarization of the pure-mode solution (red online) and of the mixed-mode solution (green online), and $I_{1 y}, I_{2 y}$ calculated numerically by integrating the rate equations (dots, see text for details) with the following parameters: $k=300 \mathrm{~ns}^{-1}, \mu=2, \alpha=3, \gamma_{N}=0.5 \mathrm{~ns}^{-1}, \tau=3 \mathrm{~ns}$, $\beta=0.04, \epsilon_{x x}=0.01, \epsilon_{x y}=0.015, \epsilon_{y x}=0.02, \epsilon_{y y}=0.025, \delta=-3$ $\operatorname{rad} \mathrm{GHz}$, and $\beta_{s p}=10^{-5} \mathrm{~ns}^{-1}$. In (a), the coupling strength $\eta$ gradually increases while in (b), it gradually decreases. the critical point where middle and low branches merge, which occurs for large $\eta$, is not shown).

The numerical simulations show that the mixed-mode solution is stable for small values of $\eta$ and destabilizes through a Hopf bifurcation at $\eta \sim 38 \mathrm{~ns}^{-1}$ [Fig. 1(a)] to a time-periodic pulsing dynamics in the two polarized modes (in Fig. 1, $I_{x}$ is not shown). Further increase of the coupling strength leads to a sequence of bifurcations to more complicated waveforms until the numerical solution switches to the pure mode at $\eta \sim 78 \mathrm{~ns}^{-1}$.

As can be expected, hysteresis occurs when the coupling strength is decreased [Fig. 1(b)]: the pure mode is numerically stable until $\eta \sim 50 \mathrm{~ns}^{-1}$, where an abrupt switching occurs, back to a pulsating dynamics in both polarizations.

The periodic or chaotic attractor that develops from the mixed mode coexists with the pure-mode solution and possibly also with other attractors: As can be seen in the bifurcation diagrams in the forward direction [Fig. 1(a)] and in the backward direction [Fig. 1(b)], the attractors are not the same.

Some analytical insight can be gained by an inspection of the $10 \times 10$ matrix that determines the linear stability of the pure-mode solution. Because of the block-diagonal form of the stability matrix, we can extract one eigenvalue that controls the stability of the pure mode. This real eigenvalue is proportional to the carrier density and more specifically to $g_{x}-1$ of the injected laser (that with $E_{x}=0$ ). We found that, for $g_{x}-1<0\left(g_{x}-1>0\right)$, the real eigenvalue is negative (positive). The change of sign occurs at the value of the coupling strength $\eta_{c}$ that can be computed from Eq. (8) with $I_{y}$ being a solution of the quadratic equation

$$
\epsilon_{x y}\left(1+\epsilon_{y y}\right) I_{y}^{2}+\left[1+\epsilon_{x y}+\epsilon_{y y}(1-\mu)\right] I_{y}+(1-\mu)=0 .
$$

Depending on the parameters, the change of sign of this eigenvalue occurs either at the upper or at the lower branch of the pure-mode $S$ curve; for the parameters chosen here, it occurs at the upper branch at $\eta_{c}=50.13 \mathrm{~ns}^{-1}$. The other eigenvalues that determine the stability of the pure mode are those of the reduced matrix that contain delay terms and can not be calculated analytically. Based on the analysis of $g_{x}-1$ as a function of $\eta$, we find that, for $\eta<\eta_{c}$, the pure mode is unstable and any other bifurcation that may emerge from the pure mode in this range will also be unstable. However, for $\eta>\eta_{c}$, we can not make, analytically, any statement about the stability of the pure mode, as it depends on the other eigenvalues. As indicated before, we find numerically that trajectories starting with initial conditions close to the puremode solution are unstable for coupling values of $\eta \lesssim 50 \mathrm{~ns}^{-1}$ as predicted also by the analysis Fig. 1(b).

To summarize, and as will be discussed in detail in the next section, in Fig. 1 the labels I, II, and III indicate the three regions of qualitatively different behavior. In regions I and III, there are transient oscillations toward the stable steady states: In region $\mathrm{I}$, for $\eta<38 \mathrm{~ns}^{-1}$, the mixed mode is stable, while in region III, for $\eta>50 \mathrm{~ns}^{-1}$, the pure mode is stable. In region II, for $38 \mathrm{~ns}^{-1}<\eta<50 \mathrm{~ns}^{-1}$, neither of them is stable and there are various types of antiphased oscillations of the $x$ and $y$ polarizations, including the particular case of regular square waves. 


\section{NUMERICAL RESULTS}

\section{A. Square-wave polarization switching}

The model equations were integrated with initial conditions such that the two lasers are off $\left[E_{x, i}(t), E_{y, i}(t)\right.$ at the noise level in the time interval $-\tau \leqslant t \leqslant 0$ and $\left.N_{i}=0, i=1,2\right]$, and parameters such that, without coupling, the two lasers emit the $x$ polarization. Unless otherwise explicitly stated, the laser parameters are $k=300 \mathrm{~ns}^{-1}, \mu=2, \alpha=3, \gamma_{N}=0.5 \mathrm{~ns}^{-1}$, $\beta=0.04, \epsilon_{x x}=0.01, \epsilon_{x y}=0.015, \epsilon_{y x}=0.02, \epsilon_{y y}=0.025$, $\delta=-3 \mathrm{rad} / \mathrm{ns}, \beta_{s p}=10^{-4} \mathrm{~ns}^{-1}$, and the coupling parameters are $\eta=50 \mathrm{~ns}^{-1}, \tau=3 \mathrm{~ns}$. In the following figures, the intensity of the $x$ polarization will be represented by a thick line (red online), and the intensity of the $y$ polarization by a thin line (blue online).

Particular combinations of the gain saturation coefficients and the linear loss anisotropy can result in polarization bistability $[37,38]$. However, here we chose parameter values such that, for the coupled lasers, we observe numerically waveforms similar to those seen experimentally [26]. We verified that, for these parameters and without coupling, the lasers emit the $x$ polarization. For the solitary lasers, when increasing the injection current parameter $\mu$, we did not see any polarization instability or switching.
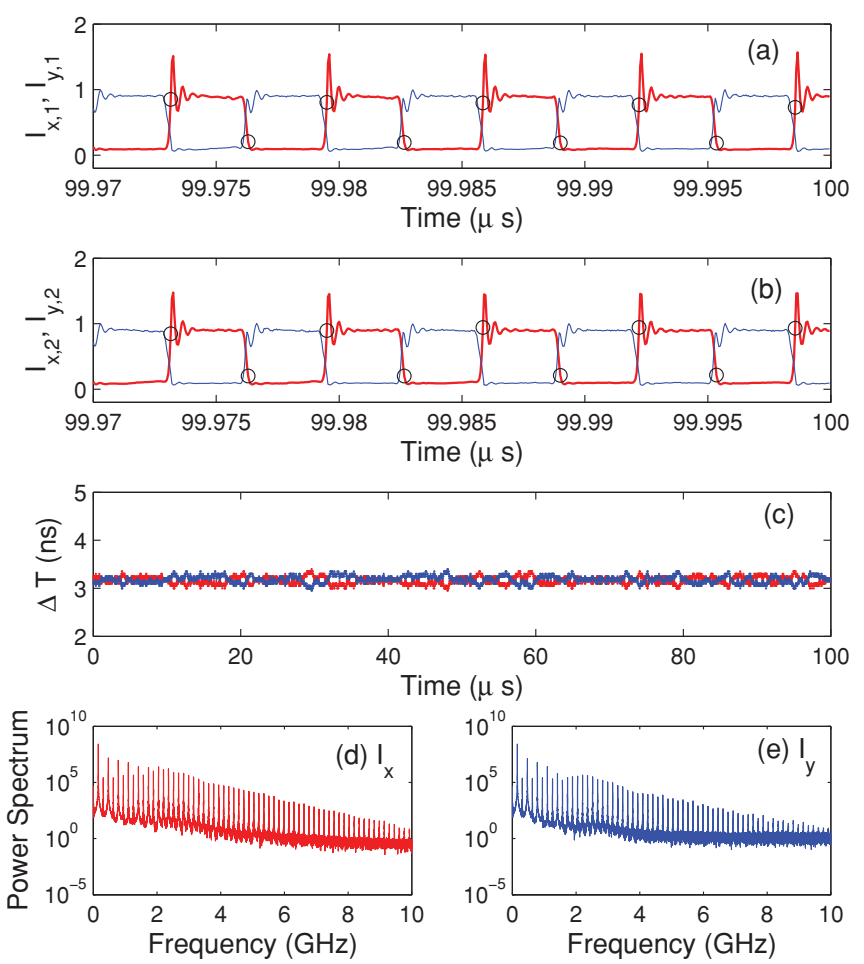

FIG. 2. (Color online) Numerically stable square-wave switching. (a), (b) Time traces of the $x$ (thick line, red online) and $y$ (thin line, blue online) polarizations of laser 1 (a) and of laser 2 (b). Notice that the lasers emit the same polarization and switch simultaneously. In (a) and (b), the circles indicate the switching times $t_{i}$. In (c), the time intervals spend in each polarization $\Delta t=t_{i}-t_{i-1}$ are plotted vs time for laser 1 . The switchings are regular, and the switching time is slightly larger than $\tau=3 \mathrm{~ns}$. (d), (e) Intensity power spectrum of the $x$ and $y$ polarizations of laser 1 . The coupling strength is $\eta=50$ $\mathrm{ns}^{-1}$, and other parameters are as indicated in the text.
As shown in Figs. 2(a) and 2(b), for these parameters and with mutual coupling, the lasers display square waves that are numerically stable, in the sense that they persist with a simulation time up to $200 \mu \mathrm{s}$. In the time traces of Figs. 2(a) and 2(b), one can notice that both the $x$ mode and the $y$ mode remain "on" (i.e., above the noise level) all the time in the two lasers; however, the $x$ and $y$ modes switch between on states (where $I_{x, i}, I_{y, i} \simeq \mu-1, i=1,2$ ) and "almost off" states (where $I_{x, i}, I_{y, i} \simeq 0, i=1,2$ ). The switching occurs simultaneously in the two lasers, and there are weak relaxation oscillations when the intensity approaches the on state.

Figure 2(c) displays, for laser 1, the sequence of switching intervals, i.e., the intervals in which one linear polarization is emitted, and one can see that the switchings occur regularly, with a periodicity slightly larger than $\tau$ [note the different time scale in (c) with respect to panels (a) and (b)]. The duration of the on state and of the off state is about half the period of the square waves for both lasers. However, the switching interval shows a randomness about its average [Fig. 2(c)] that is entirely due to the spontaneous emission noise included in the stochastic rate equations. The integration of the deterministic rate equations shows that the switching interval is constant in time within the resolution of the calculation. Finally, we point out that the rf spectra of the intensities [Figs. 2(d) and 2(e)] have a large number of harmonics at frequencies of the delay as expected for square waves.

\section{B. Bifurcation scenarios}

The stability of the regular square waves depends on several parameters such as the coupling strength $\eta$, the detuning between the two polarizations $\delta$, and their different losses $\beta$.

First, we discuss the influence of the coupling strength on the basis of the bifurcation diagram shown in Fig. 1. In the region labeled I, the laser system exhibits a steady-state mixed-mode solution, i.e., stationary intensities in both $x$ and $y$-polarization modes for both lasers and, moreover, the solution is symmetric when exchanging the lasers: $I_{1 x}=I_{2 x}$ and $I_{1 y}=I_{1 y}$. Figure 3 shows the transient dynamics to the steady-state two-mode solution. As the coupling increases, this mixed-mode steady-state solution becomes unstable at a Hopf bifurcation diagram, and the laser enters the region
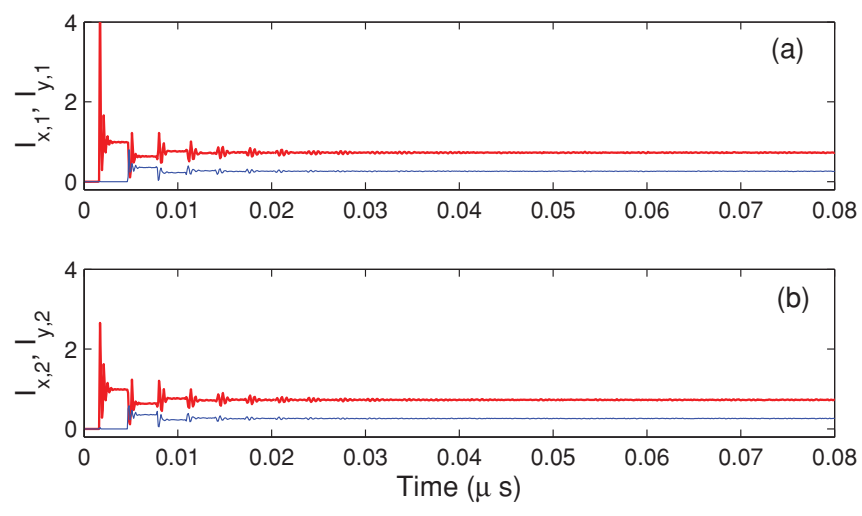

FIG. 3. (Color online) Transient dynamics toward a mixed-mode solution. Parameters are as in Fig. 2 but with weaker coupling strength $\eta=30 \mathrm{~ns}^{-1}$. 

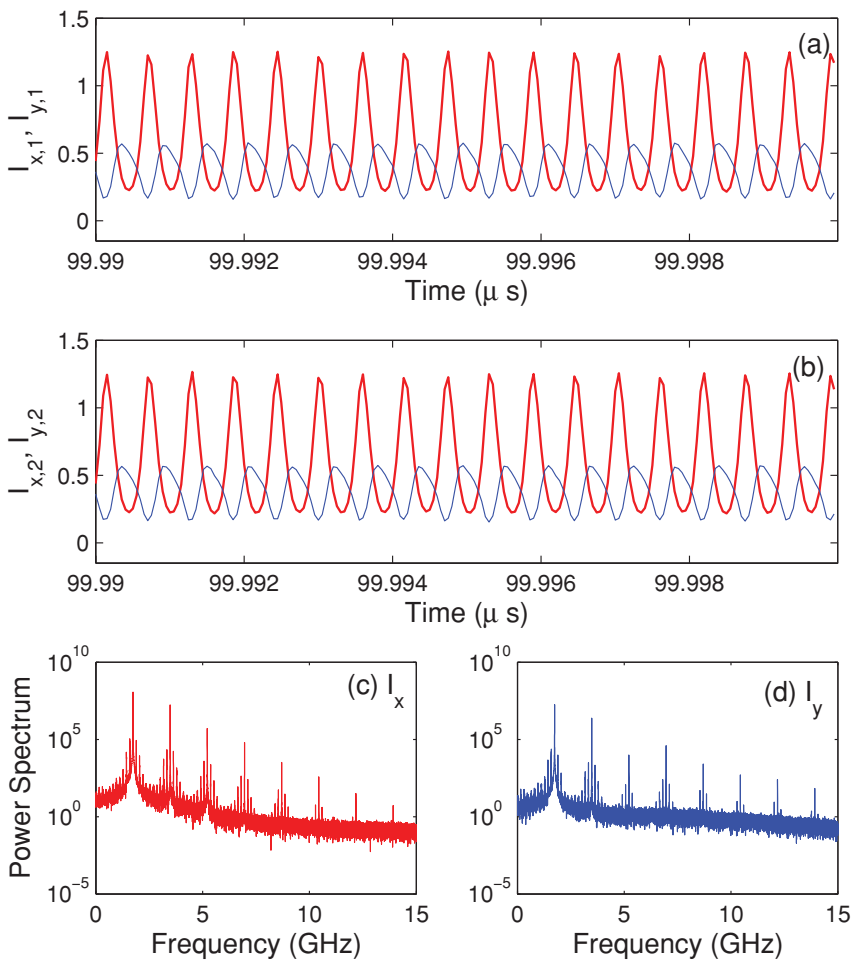

FIG. 4. (Color online) Symmetric antiphased sinusoidal oscillations $\eta=40 \mathrm{~ns}^{-1}$ and other parameters are as in Fig. 2. (a), (b) Intensity of the $x$ and $y$ polarizations in lasers 1 and 2; (c), (d) intensity power spectrum of the $x$ and $y$ polarizations of laser 1 .

labeled II where the intensities of both lasers start pulsating at a frequency much faster than the inverse of the time delay, and close to or related to the relaxation oscillation frequency. An example of such a time-periodic dynamics in the two modes is shown in Fig. 4. The pulsating dynamics keeps the symmetry property of the mixed-mode solution, i.e., the laser $2 x$ - and $y$-mode intensity dynamics are the same as those of laser 1 mode intensities.

For increasing coupling strengths, the laser exhibits a large variety of pulsating dynamics and irregular switchings between polarization modes. Solutions may be different depending on the noise level in the simulations and depending on the initial conditions, either starting from a mixed-mode solution or from a pure-mode solution. The solutions may be symmetric when exchanging lasers 1 and 2 or, by contrast, nonsymmetric with different time traces for all mode intensities. An example of such typical waveform is plotted in Fig. 5. When comparing to Fig. 4, the intensity dynamics now shows features on a slower time scale, close to twice the time delay. It is worth mentioning, as an example of multistability, that for the same value of $\eta=48 \mathrm{~ns}^{-1}$, there is another symmetric and regular pulsating dynamics in the mode intensities, at a time period close to twice the time delay.

As the coupling strength is increased further, the dynamics is dominated by time-periodic and irregular, symmetric and nonsymmetric pulsations, although it starts seeing the influence of the coexistence with a stable pure-mode steadystate solution. For $\eta=50 \mathrm{~ns}^{-1}$, we have shown in Fig. 2 a regular and symmetric square-wave switching solution at the period of twice the time delay. For slightly smaller
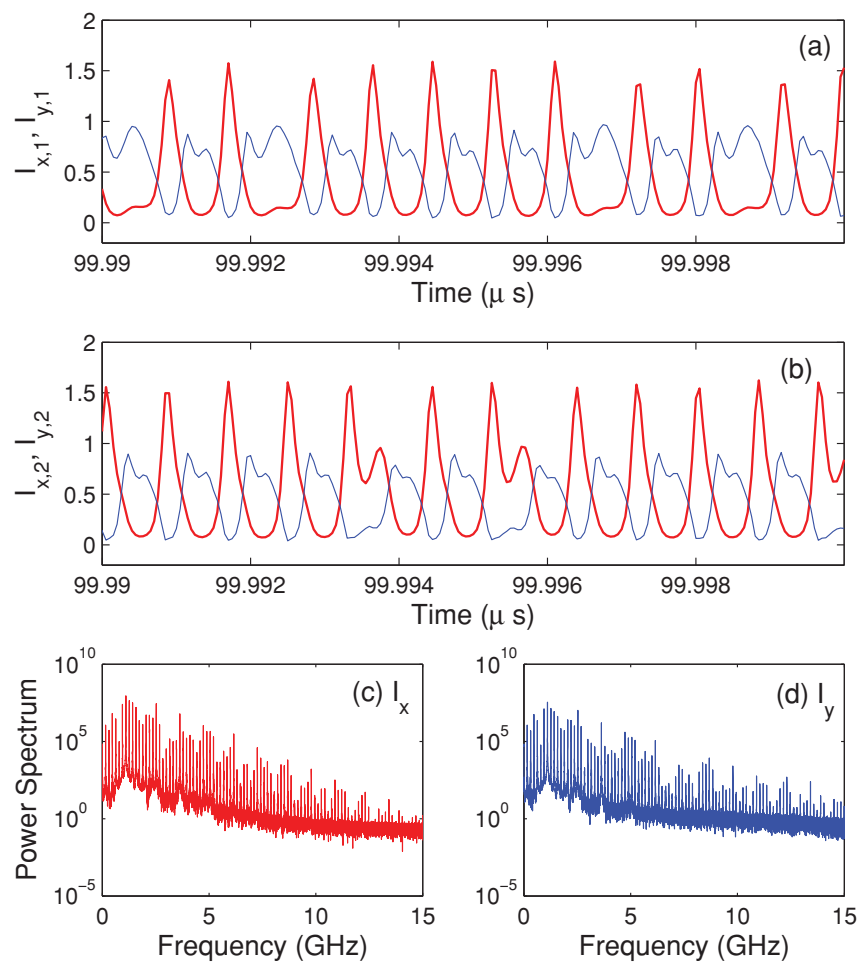

FIG. 5. (Color online) Nonsymmetric antiphased pulselike oscillations $\eta=48 \mathrm{~ns}^{-1}$ and other parameters are as in Fig. 2. (a), (b) Intensity of the $x$ and $y$ polarizations in lasers 1 and 2; (c), (d) intensity power spectrum of the $x$ and $y$ polarizations of laser 1 .

coupling strength $\left(\eta=49.8 \mathrm{~ns}^{-1}\right)$, we illustrate in Fig. 6 another square-wave switching dynamics in the polarization dynamics, but this one being irregular and nonsymmetric upon the exchange of both lasers. For slightly larger coupling strength $\left(\eta=50.2 \mathrm{~ns}^{-1}\right)$, we observe in Fig. 7 that the lasers emit light with orthogonal polarization, and there are regular pulses with periodicity slightly larger than $2 \tau$ in the other polarization [e.g., in Fig. 7, we can see that laser 1 emits the $y$ polarization with regular pulses in the $x$ polarization]. One can also notice that the lasers do not emit the pulses simultaneously, but alternate, such that laser 1 emits a pulse at time $t$, and laser 2 , at time $\sim t+\tau$. It is worth mentioning that the gradual change in the intensity power spectra in Figs. 4, 5, 6, and 2 suggests that the regular square waves seen in Fig. 2 (that actually occur only in a very narrow region of $\eta$ values) arise due to a kind of frequency "locking."

For even stronger coupling, when entering the region III, the square-wave switching becomes a transient dynamics toward the "pure-mode" solutions discussed in the preceding section, which are symmetric with respect to exchanging the lasers.

A typical transient is presented in Fig. 8, where one can see that, after the transient, for laser 1 we have $I_{1, x}=\mu-1=1$, $I_{1, y}=0$, and for laser $2, I_{2, x}=0, I_{2, y} \neq 0$. Since $I_{2, x}=0$, laser 1 does not receive optical injection (the laser ending up in this state will be referred to as the "solitary laser"). As for laser 2 , it receives injection from laser 1 that is strong enough to turn on the $y$ mode and to suppress the natural lasing mode $x$ (the laser ending up in this state will be referred to as the "injected laser"). 

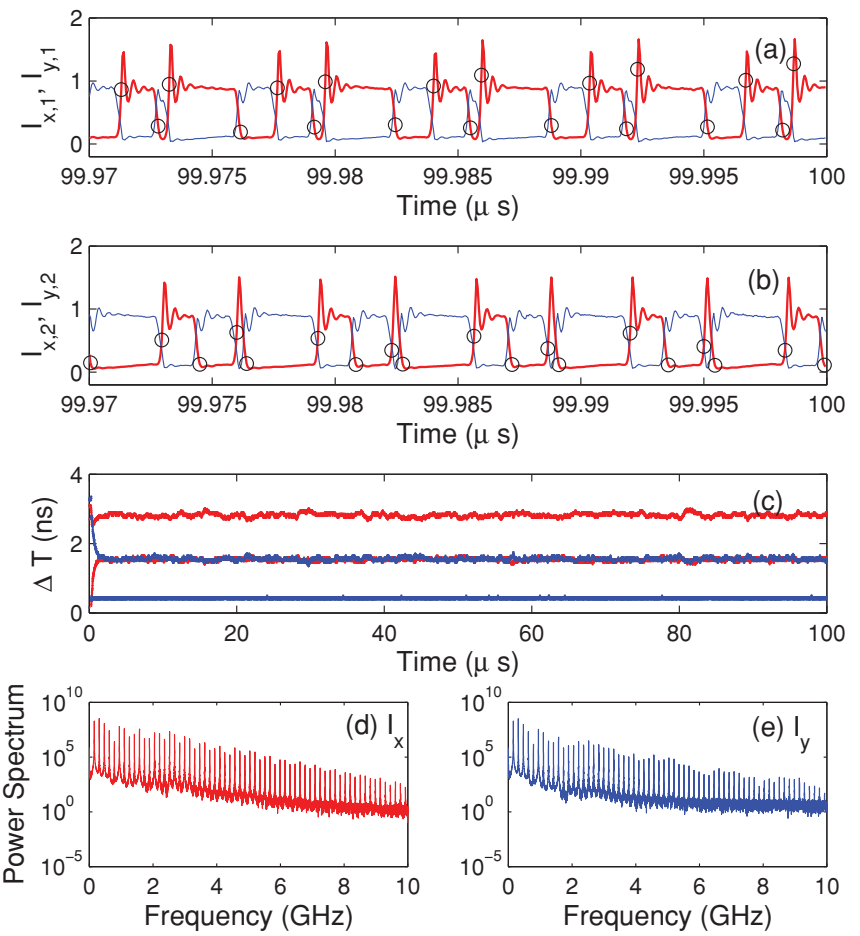

FIG. 6. (Color online) Nonsymmetric and irregular squarelike switching $\eta=49.8 \mathrm{~ns}^{-1}$ and other parameters are as in Fig. 2. (a), (b) Intensity of the $x$ and $y$ polarizations in lasers 1 and 2. In (a) and (b), the circles indicate the switching times $t_{i}$. In (c), the time intervals spent in each polarization, $\Delta t=t_{i}-t_{i-1}$, are plotted vs time for laser 1. (d), (e) Intensity power spectrum of the $x$ and $y$ polarizations of laser 1 .

We conclude this section by discussing the influence of different laser parameters on the stability of the regular square-wave switchings shown in Fig. 2. As mentioned before, this type of waveform occurs in a narrow parameter region and if, for example, the lasers have slightly different injection currents, instead of regular switching, we observe pulsing waveforms as those shown in Fig. 7.

\section{Analysis of the stochastic transient time}

For parameters where the polarization antiphased oscillations are a transient dynamics, we studied the duration of the transient and found that it is a stochastic quantity, determined by the random initial conditions (i.e., the noisy initial values of $E_{x, i}$ and $E_{x, i}$ in the time interval $-\tau \leqslant t \leqslant 0$ ) and on the spontaneous emission noise in the rate equations.

Figures 9(a) and 9(b) display in the parameter space $(\beta, \eta)$ the mean transient time $\left\langle T_{s q}\right\rangle_{s}$ and its normalized standard deviation $\sigma_{s q} /\left\langle T_{s q}\right\rangle_{s}$. Here, $\langle\cdots\rangle_{s}$ indicates an "ensemble average" over 50 stochastic trajectories that were simulated, with different noise realizations, for each set of parameters $(\beta, \eta)$.

For each stochastic trajectory, the duration of the transient was determined by analyzing the oscillations of the intensity of the $x$ polarization in the two lasers. Specifically, we calculated the normalized dispersion of the intensity oscillations $\sigma_{i} /\left\langle I_{x, i}\right\rangle_{t}$, where $\langle\cdots\rangle_{t}$ indicates an average over a time window of length $5 \tau$ that moves along the time series.
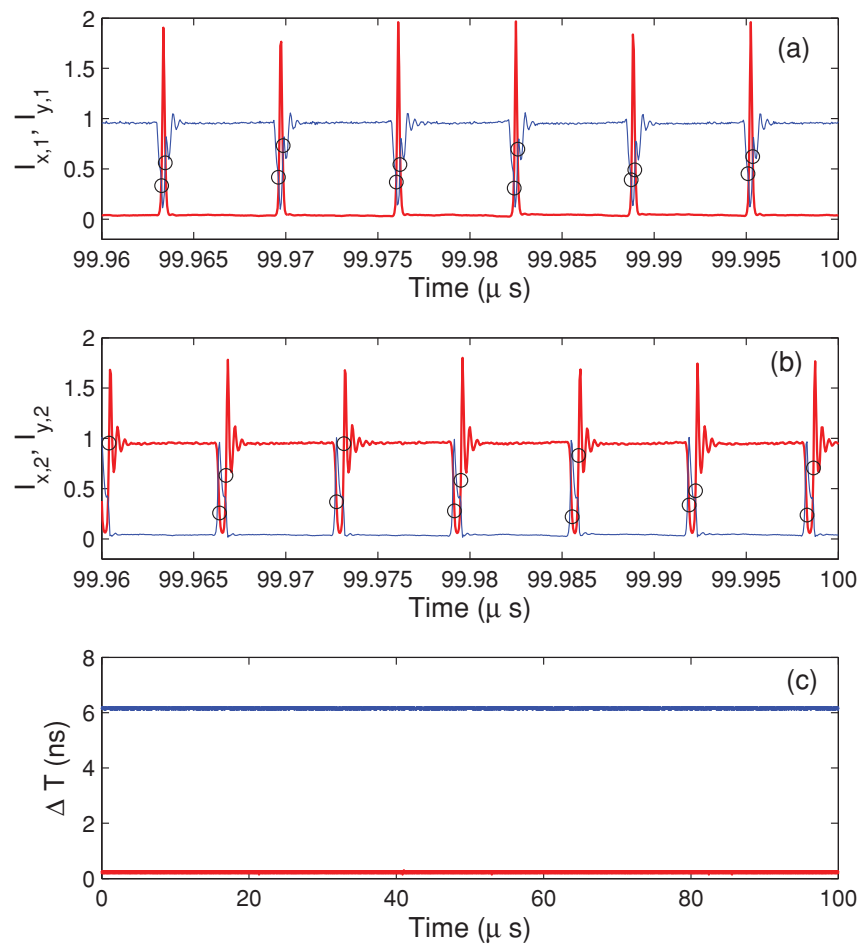

FIG. 7. (Color online) Nonsymmetric regular pulsing behavior $\eta=50.2 \mathrm{~ns}^{-1}$ and other parameters are as in Fig. 2. (a), (b) Intensity of the $x$ and $y$ polarizations in lasers 1 and 2. In (a) and (b), the circles indicate the switching times $t_{i}$. In (c), the time intervals spent in each polarization $\Delta t=t_{i}-t_{i-1}$ are plotted vs time for laser 1 .

Whenever $\sigma_{1} /\left\langle I_{x, 1}\right\rangle_{t}$ or $\sigma_{2} /\left\langle I_{x, 2}\right\rangle_{t}$ were small enough [see panel (d) in Fig. 8 that display the typical time evolution of this quantifier], we considered that the dynamics reached an asymptotic behavior and thus determined the duration of the transient. Clearly, the duration of the transient is a stochastic quantity that depends on the random initial conditions and the noise in the trajectory. For each trajectory, the maximum simulation time was $50 \mu \mathrm{s}$ and, therefore, this is the longest transient time that we were able to calculate.

In Fig. 9(a), that displays the logarithm of transient time, one can see that the transient is either short (top-left and bottom-right corners) or equal to the maximum simulation time (white center region). In the regions where the transient finishes, the asymptotic state is in a mixed-mode solution (top-left corner) or in a pure-mode solution (bottom-right corner), while the white region in-between corresponds to the antiphased polarization oscillations. In Fig. 9(b), one can appreciate that the transient times to the mixed-mode and to the pure-mode solutions have different characteristics: When the pure-mode solution is reached, the duration of the transient has a large deviation from its mean value (being mainly a stochastic quantity), revealed by the large normalized dispersion $\sigma_{s q} /\left\langle T_{s q}\right\rangle$. On the contrary, when a mixed-mode solution is reached, the transient time has a small deviation from its mean value (being mainly a deterministic quantity), and the value of $\sigma_{s q} /\left\langle T_{s q}\right\rangle$ is small.

Figures 9(c) and 9(d) display the average largest intensity of the $x$ polarization and of the $y$ polarization, computed as 

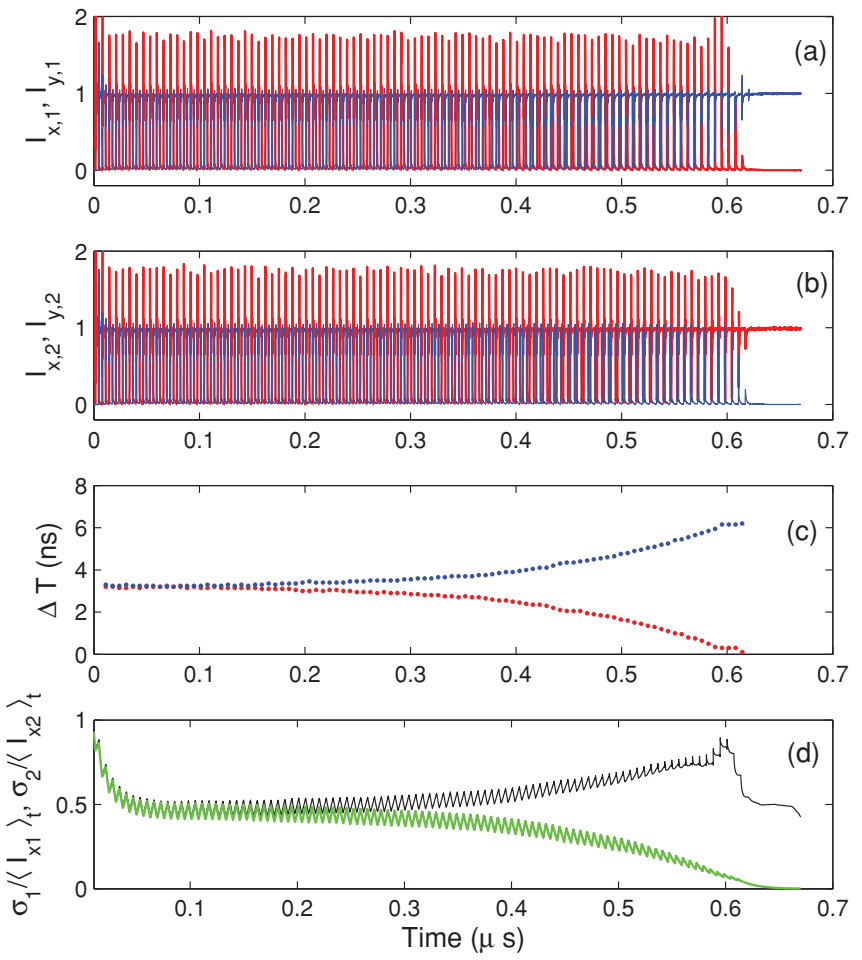

FIG. 8. (Color online) Transient square waves. Parameters are as in Fig. 2, but with larger coupling strength $\eta=51 \mathrm{~ns}^{-1}$. (a), (b) Intensity of the $x$ and $y$ polarizations in lasers 1 and 2. (c) Switching time intervals of laser 1 . It can be noticed that toward the end of the transient, the intervals spent in one polarization $(x)$ gradually increase while the intervals spent in the orthogonal polarization gradually decrease. (d) Time variation of the normalized standard deviation $\sigma_{i} /\left\langle I_{x, i}\right\rangle_{t}$ of the intensities of the $x$ polarization in the two lasers (laser 1: thin black line, laser 2: thick line, green online). $\sigma_{i} /\left\langle I_{x, i}\right\rangle_{t}$ are calculated over a moving time window of length $5 \tau$. One can notice that when the transient finishes, laser 1 reaches stable continuous wave emission on the $x$ polarization, and $\sigma_{1} /\left\langle I_{x, 1}\right\rangle_{t} \sim 0$.

follows:

$$
\begin{aligned}
& \left\langle I_{x}\right\rangle=\left\langle\max \left(\left\langle I_{x, 1}\right\rangle_{t},\left\langle I_{x, 2}\right\rangle_{t}\right)\right\rangle, \\
& \left\langle I_{y}\right\rangle=\left\langle\max \left(\left\langle I_{y, 1}\right\rangle_{t},\left\langle I_{y, 2}\right\rangle_{t}\right)\right\rangle,
\end{aligned}
$$

where $\langle\cdots\rangle_{t}$ denotes a time average and $\langle\cdots\rangle$ denotes an an average over the 50 stochastic trajectories.

In Figs. 9(c) and 9(d), one can distinguish two different regions: For large $\beta$ and small $\eta$ (top left corner), $\left\langle I_{x}\right\rangle$ is close to 1 and $\left\langle I_{y}\right\rangle$ is close to 0 , indicating that in the asymptotic state the intensity of the $x$ polarization ( $y$ polarization) is large (small) in both lasers. On the opposite corner, both $\left\langle I_{x}\right\rangle$ and $\left\langle I_{y}\right\rangle$ are close to 1 , indicating that in the asymptotic state one laser emits the $y$ polarization (the injected laser), while the other emits the $x$ polarization (the solitary laser). In-between these two regions, there is a region where the largest intensities of the $x$ and $y$ modes are similar. This region corresponds, in Fig. 9(a), to the region where the transient time is equal to the maximum simulation time (i.e., is the region where antiphased oscillations and square-wave regular switching occur).

Another relevant parameter determining the duration of the transient time is the frequency detuning between the $x$ and $y$ polarizations $\delta$. Figure 10 displays the mean transient time,

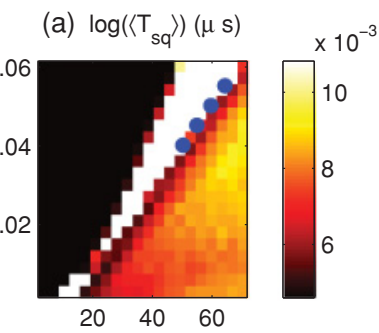

(c) $\left\langle\left.\right|_{\mathrm{x}}\right\rangle$

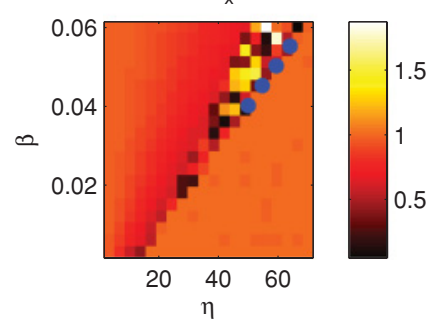

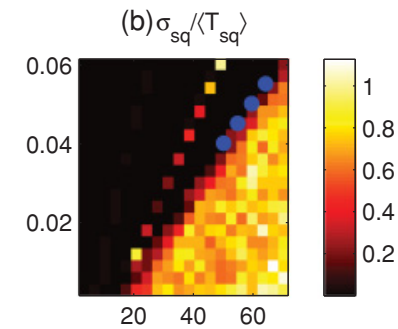

$(d)\left\langle I_{y}\right\rangle$

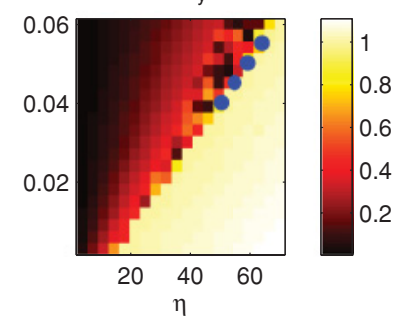

FIG. 9. (Color online) Phase diagram in the parameter space (cavity-loss difference $\beta$, coupling strength $\eta$ ). Logarithm of the average transient time (a), normalized dispersion of the transient time (b), the average largest intensity of the $x$ polarization (c), and of the $y$ polarization (d) (see text for details). In each caption, the dots (blue online) indicate parameters where we have seen regular square-wave switching, as that shown in Fig. 2.

its normalized dispersion, and the averaged largest intensities $\left\langle I_{x}\right\rangle$ and $\left\langle I_{y}\right\rangle$ in the parameter plane (frequency detuning, coupling strength). Again one can distinguish two regions, one for low coupling, in which the average transient time is short, its normalized dispersion close to $0,\left\langle I_{x}\right\rangle \simeq 1$, and $\left\langle I_{y}\right\rangle \simeq 0$ (mixed-mode solution); for strong coupling, the average (a) $\log \left\langle T_{s q}\right\rangle(\mu s)$

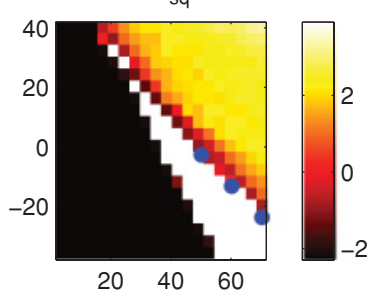

(c) $\left\langle I_{x}\right\rangle$

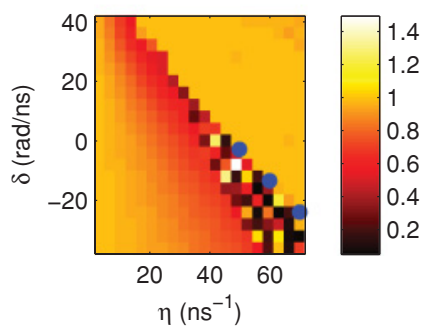

(b) $\sigma_{\mathrm{sq}} /\left\langle\mathrm{T}_{\mathrm{sq}}\right\rangle$

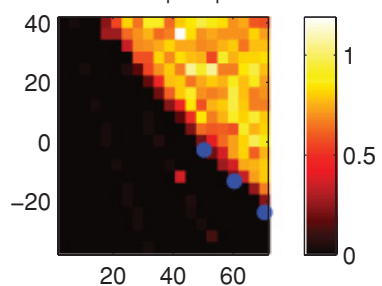

(d) $\left\langle I_{\mathrm{y}}\right\rangle$

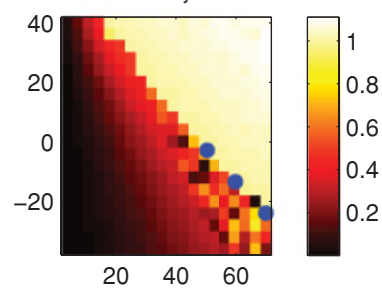

FIG. 10. (Color online) Phase diagram in the parameter space (mode frequency detuning $\delta$, coupling strength $\eta$ ). Logarithm of the average transient time (a), normalized dispersion of the transient time (b), the average largest intensity of the $x$ polarization (c), and of the $y$ polarization (d) (see text for details). In each caption, the dots (blue online) indicate parameters where we have seen regular squarewave switching, as that shown in Fig. 2. 
transient is longer, its dispersion is larger, and both $\left\langle I_{x}\right\rangle \simeq 1$ and $\left\langle I_{y}\right\rangle \simeq 1$ (pure-mode solution). In-between these two regions, there is a region where the transient time equals the maximum simulation time. In this region, for most parameters, antiphased oscillations occur, but also regular square-wave switching is seen for specific combinations of parameter values. In certain regions, we also have found coexistence of antiphased oscillations with nearly constant two-mode output (a mixed-mode solution), and this coexistence results in large values of the normalized dispersion of the transient time [small red region embedded in the black area in Fig. 10(b)].

\section{EXPERIMENTS}

The experimental apparatus used to investigate the dynamics of the orthogonal mutual coupling system is shown in Fig. 11. The dynamical system itself consists of a $U$-shaped configuration (red online), with two temperature-stabilized EELs (LD1 and LD2) and optical components to control polarization rotation, coupling strength, and beam steering. The lasers are model SDL-5401 MQW devices, each with nominal wavelength of $818 \mathrm{~nm}$ and current threshold of $18.5 \mathrm{~mA}$. Each laser beam is collimated by a lens (CL), encounters a beam sampler (BS), and then passes through a Faraday rotator (ROT) with linear output polarizer (OP) oriented at $45^{\circ}$, which thus allows only the TE mode to pass through going forward and extinguishes any TM output or reflections. After passing through a rotatable polarizer (POL) used to control coupling strength, the beam enters a second output polarizer and Faraday rotator. The resulting beam, now rotated $90^{\circ}$ in polarization, is injected into the other laser. All unlabeled components in the schematic diagram are high-reflectivity mirrors.

The beam paths for detection (thin line, blue online) are also shown in Fig. 11. The plate beam samplers (BS) are oriented at near-normal incidence to minimize polarization dependence of reflectivity; they divert 5\% of each beam to the detection branches. Polarizing beam-splitter cubes (PBS) separate the light into $x$ - and $y$-polarization components, which then are attenuated as needed with neutral density filters (ND) before striking photodetectors (PD) with 8.75-GHz bandwidth. The detected signals are amplified by $23 \mathrm{~dB}$ using wideband $(10 \mathrm{kHz}-12 \mathrm{GHz})$ ac amplifiers (AMP) and are captured

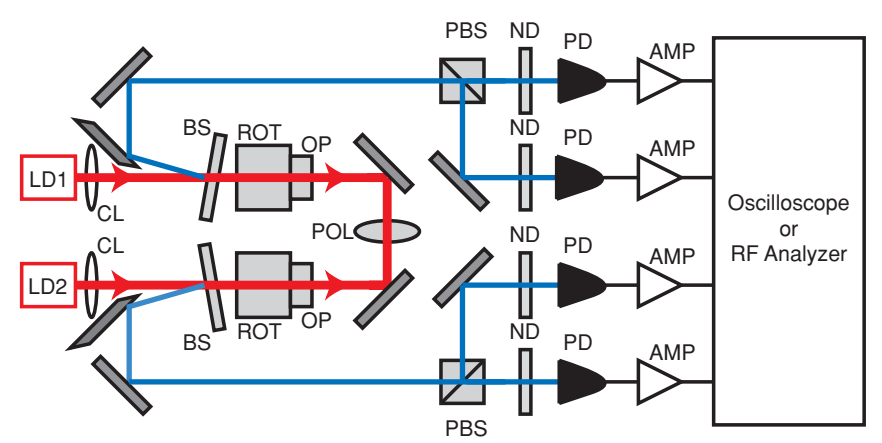

FIG. 11. (Color online) Schematic diagram of experimental apparatus. Heavy line (red online) shows beam path of dynamical system; thin line (blue online) indicates beam paths for detection. All definitions of abbreviations are in the text.
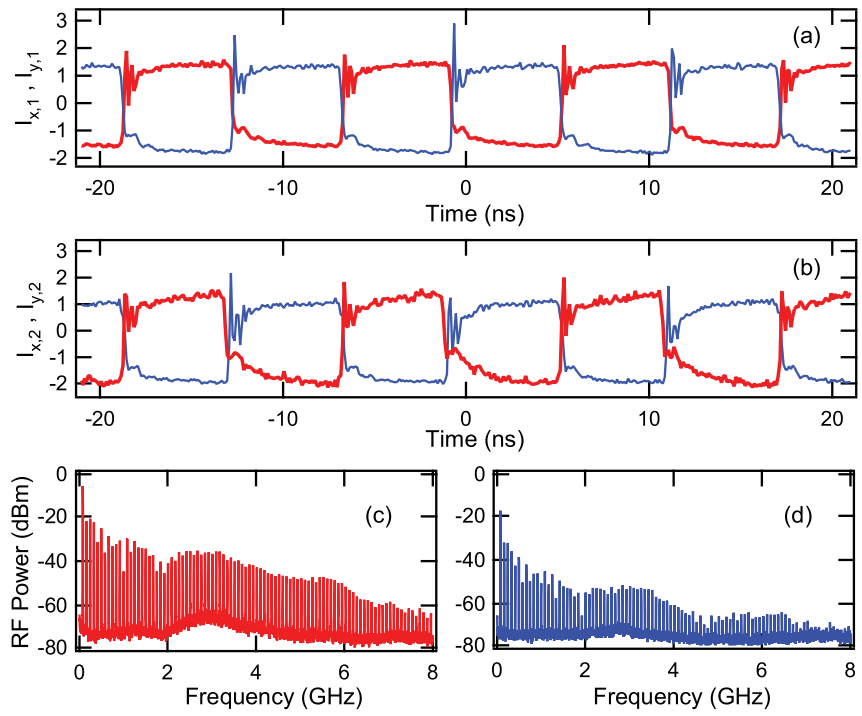

FIG. 12. (Color online) Experimentally observed square-wave dynamics. (a), (b) Time traces of the $x$ (thick line, red online) and $y$ (thin line, blue online) polarizations of laser 1 (a) and laser 2 (b). (c), (d) Intensity power spectra of the $x$ and $y$ polarizations of laser 1 . Operating conditions are noted in the text.

and displayed on either a 6-GHz digital storage oscilloscope or radiofrequency spectrum analyzer. It is important to note that the detectors and amplifiers are strictly ac coupled, and therefore can not be used to determine absolute power levels.

Experimental observations from this setup agree well with many numerical results presented in the previous sections. Figure 12 shows polarization-resolved time series and rf spectra for square waveforms, similar to those shown previously in Fig. 2. Figures 12(a) and 12(b) show time traces of the intensities of both modes of both lasers. The modes within each laser are in antiphase, but the two lasers emit in the same polarization and exhibit switching simultaneously. Figures 12(c) and 12(d) display the corresponding power spectra for the $x$ and $y$ polarizations of laser 1, with many higher harmonics, and a fundamental close to the inverse of $2 \tau$. For these data, both lasers were pumped at 2.10 times the threshold current; the cavity length was $1.67 \mathrm{~m}$, corresponding to $\tau=5.57 \mathrm{~ns}$, and the one-way cavity transmission was $48.7 \%$.

The symmetric waves in Fig. 12 change smoothly to asymmetric square waves if the coupling is changed. Figure 13 illustrates this case, where all experimental conditions remain the same as in Fig. 12, except for a reduction in the cavity transmission to $46.6 \%$. In the polarization-resolved intensity time traces for each laser [Figs. 13(a) and 13(b)], the plateaus of the $x$ and $y$ components now are different in duration, but still in antiphase within each laser. In addition, they now are nonsymmetric under exchange of the lasers. Figures 13(c) and 13(d) show the rf spectra for the two components of laser 1 ; each still has the same fundamental frequency as the square case, but the harmonic content is changed.

Due to the ac detection used in the experiments, we can not identify if these square waves correspond to the numerical solution where both polarization modes are always on, or the transient solution where the mode which is off emits only at the noise level. However, the experimental waves are stable 

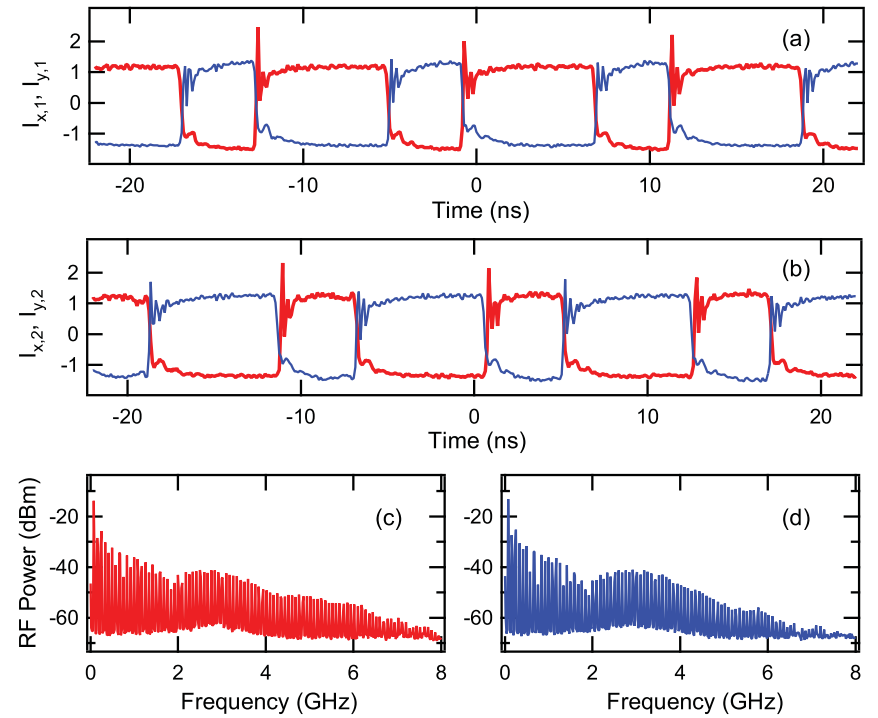

FIG. 13. (Color online) Experimentally observed asymmetric and nonsymmetric square-wave dynamics resulting from change in coupling strength. (a), (b) Time traces of the $x$ (thick line, red online) and $y$ (thin line, blue online) polarizations of laser 1 (a) and laser 2 (b). (c), (d) Intensity power spectra of the $x$ and $y$ polarizations of laser 1. Operating conditions are noted in the text.

over a duration of tens of minutes, indicating that, if they are transients, there must be a stabilizing mechanism.

Figure 14 illustrates a bifurcation sequence as a function of increasing coupling strength. For visual clarity, intensity time series in one mode of one laser only are shown. For these data, the distance between lasers is $93.5 \mathrm{~cm}$, so $\tau=3.12 \mathrm{~ns}$; both lasers are pumped at twice threshold. The sequence of coupling

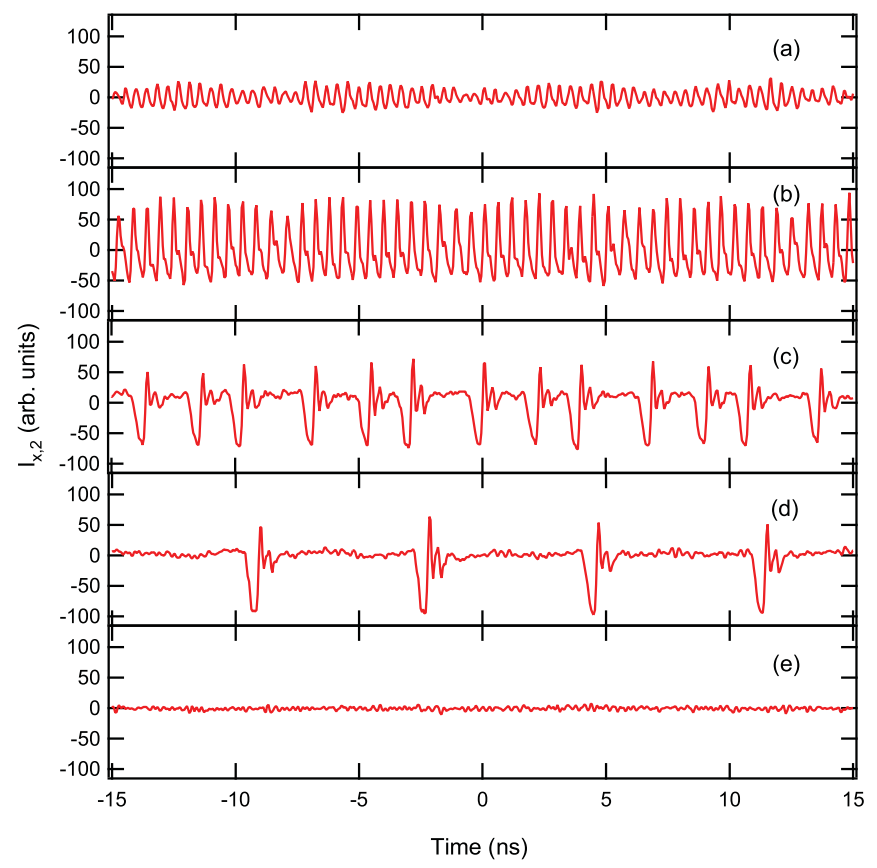

FIG. 14. (Color online) Experimental bifurcation sequence as a function of coupling strength, shown as time traces of $x$ polarization of laser 2. Coupling strength increases from top (a) to bottom (e). Operating conditions are noted in the text.

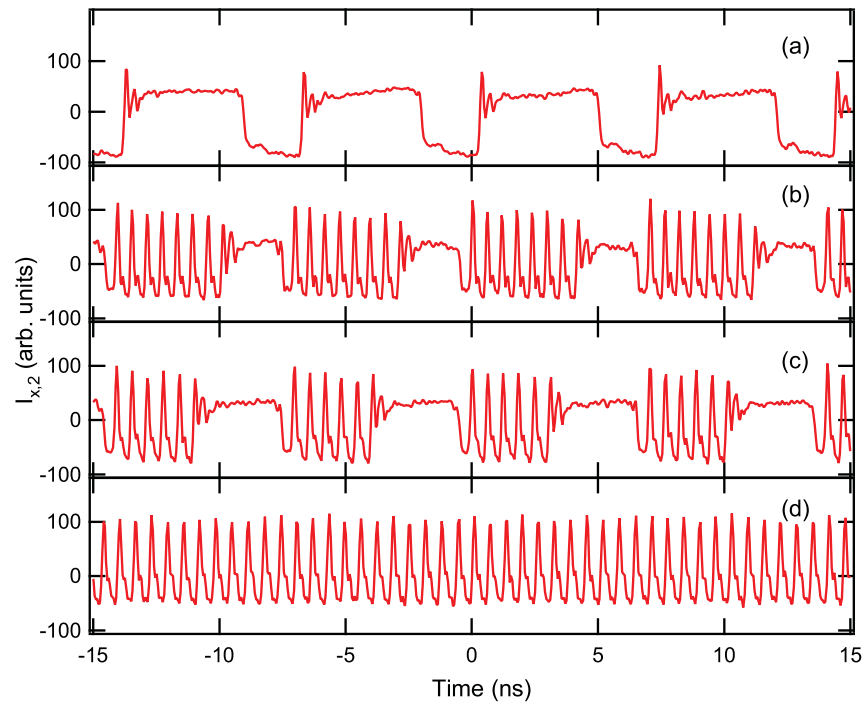

FIG. 15. (Color online) Experimental set of coexisting solutions, shown as time traces of $x$ polarization of laser 2. Operating conditions are noted in the text, and are constant for all traces displayed. Puremode steady states (not shown) also coexist with these dynamical solutions.

strengths from Figs. 14(a) though 14(e), expressed again as one-way fractional power transmission, are $1.5 \%, 13.3 \%$, $15.8 \%, 24.3 \%$, and $34.5 \%$. Figure 14(a) shows weak oscillations on a mixed-mode state, which bifurcate in Fig. 14(b) to a stronger oscillatory solution close to the relaxation oscillation frequency, similar to that shown in Fig. 4. In Fig. 14(c), there is irregular squarelike switching (compare Fig. 6), which evolves to a regular pulsing form (compare Fig. 7), and in both of these the frequency is now governed by the slower time scale of the delay. Finally, in Fig. 14(e), the system goes to a stable pure-mode solution. This sequence also agrees with theoretical predictions, as summarized in Fig. 1. Similar sequences were observed experimentally at other pump currents, as long as the values for each laser are almost the same.

Another prediction of Fig. 1 is the existence of regions of multistability involving both pulsating and steady-state dynamics. Such regions are observed experimentally, and a sample of coexisting solutions is shown in Fig. 15. Again, we show intensity time series in one mode only to illustrate. All data for this figure were captured with $\tau=3.12 \mathrm{~ns}$, power transmission of $32.8 \%$, and pumping at 2.05 times threshold. It is clear that square waves, oscillating solutions, and various mixes of the two all can coexist simultaneously. Perturbing the system or randomizing initial conditions allows one to change between solutions. In addition to the various pulsing and switching waveforms shown, both pure-mode steady states also were observed to exist stably in this region. Indeed, square-wave solutions have only been observed experimentally under conditions for which both pure modes coexist.

\section{CONCLUSIONS}

In summary, we have performed both theoretical and experimental analyses of the complex dynamics occurring in a system of two edge-emitting lasers being mutually coupled through their normally nonlasing TM $(y)$ polarizations. 
Our first goal is to characterize numerically square-wave polarization switching dynamics, similar to those recently reported experimentally [26]. Key ingredients for the observation of stable switching dynamics appear to be the frequency detuning between the two polarizations $(\delta)$ and the gain self- and cross-saturation coefficients $\left(\epsilon_{a b}\right.$ with $a, b=x$ or $y$ ). In the model employed in [26] that did not take these into account, only transient polarization switching was found.

Other more or less complex dynamics are also shown, as bifurcations from single-mode or two-mode steady-state solutions for both lasers. We found two types of square-wave switching, one in which the intensities of the two polarizations do not reach zero at any time during the oscillations, and another in which, when the intensities are at the off state, they approach zero within the limits of spontaneous emission noise. The first type of square-wave switching (Fig. 2) appears to be numerically stable and occurs in a narrow parameter region, the second type (Fig. 8) is a transient dynamics toward the puremode solution. Another important difference is that the first type of square waveform is symmetric with respect to the exchange of lasers, while the second type of square waveform is asymmetric. Also, while further research is needed to fully determine the role of noise, preliminary simulations suggest that the symmetric square waveform is unaffected by the noise strength. On the contrary, the asymmetric waveform is affected by the noise, as it is a transient dynamics toward a steady state (pure-mode solution) and the duration of the transient is a stochastic quantity that depends, not only on the initial conditions, but also on the spontaneous emission-noise realization.

The theoretical results are supported by experimental measurements, which complement the earlier observations of stable square-wave switching of Ref. [26]. Both symmetric and nonsymmetric square-wave switchings are found depending on the laser coupling strength. The bifurcation scenario leading to square-wave switching is also found experimentally when increasing the coupling strength: A Hopf bifurcation first leads to self-pulsation at the relaxation oscillation frequency, that further bifurcates to symmetric or asymmetric squarewave solutions, until the system restabilizes to a steady state for large values of the coupling strength. Moreover, the experiment also confirms the theoretical predictions of coexistence (multistability) of several dynamical solutions (steady states, self-pulsations, square-wave switchings). A detail that it is, however, not clear from the experimental data is to what the degree the intensities approach zero during the off cycle of the square waves. Since the time-series data were obtained with ac-coupled detectors, it is not possible to make a meaningful comparison with the numerical results in this respect.

We think our results could also motivate new investigations in different directions. First, a more systematic analysis of the influence of noise and of new and larger regions of the parameters would be of interest. Second, it would be interesting to compare similar polarization dynamics that may be observed in coupled VCSELs through polarized mutual optical injection, where the mode gain to loss ratio can be varied through a sweep of the laser injection current or device temperature [39].

\section{ACKNOWLEDGMENTS}

C.M. acknowledges the support of the Spanish Ministerio de Ciencia e Innovacion through Project No. FIS2009-13360C03-02, the AGAUR, Generalitat de Catalunya, through Project No. 2009 SGR 1168, EOARD Grant No. FA-8655-101-3075, and the ICREA Academia programme. D.S. acknowledges the support of the US National Science Foundation and the Lenfest Endowment. M.S. acknowledges the support of Conseil Régional de Lorraine and of COST MP0702 European Action.
[1] D. M. Kane and K. A. Shore, Unlocking Dynamical Diversity: Optical Feedback Effects on Semiconductor Lasers (Wiley, New York, 2005).

[2] J. Ohtsubo, Semiconductor Lasers: Stability, Instability and Chaos, 2nd ed. (Springer, Berlin, 2010).

[3] T. Erneux and P. Glorieux, Laser Dynamics (Cambridge University Press, Cambridge, UK, 2010).

[4] S. C. Chan, R. Diaz, and J. M. Liu, Opt. Quantum Electron. 40, 83 (2008).

[5] S. Schikora, P. Hovel, H.-J. Wunsche, E. Schöll, and F. Henneberger, Phys. Rev. Lett. 97, 213902 (2006).

[6] T. Erneux, Applied Delay Differential Equations (Springer, New York, 2000).

[7] G. Huyet, J. K. White, A. J. Kent, S. P. Hegarty, J. V. Moloney, and J. G. McInerney, Phys. Rev. A 60, 1534 (1999).

[8] D. W. Sukow, T. Heil, I. Fischer, A. Gavrielides, A. HohlAbiChedid, and W. Elsasser, Phys. Rev. A 60, 667 (1999).

[9] F. Rogister, M. Sciamanna, O. Deparis, P. Mégret, and M. Blondel, Phys. Rev. A 65, 015602 (2001).
[10] J. Houlihan, G. Huyet, and J. G. McInerney, Opt. Commun. 199, 175 (2001).

[11] C. Masoller, M. S. Torre, and P. Mandel, Phys. Rev. A 71, 013818 (2005).

[12] C. Masoller, C. Serrat, and R. Vilaseca, Phys. Rev. A 76, 043814 (2007).

[13] P. Besnard, M. L. Charès, G. Stéphan, and F. Robert, J. Opt. Soc. Am. B 16, 1059 (1999).

[14] M. Sciamanna, K. Panajotov, H. Thienpont, I. Veretennicoff, P. Mégret, and M. Blondel, Opt. Lett. 28, 1543 (2003).

[15] Y. H. Hong, P. S. Spencer, and K. A. Shore, Opt. Lett. 29, 2151 (2004).

[16] J. Houlihan, L. Lewis, and G. Huyet, Opt. Commun. 232, 391 (2004).

[17] C. Masoller and M. S. Torre, IEEE J. Quantum Electron. 41, 483 (2005).

[18] J. Paul, C. Masoller, Y. Hong, P. S. Spencer, and K. A. Shore, J. Opt. Soc. Am. B 24, 1987 (2007).

[19] C. Masoller, Phys. Rev. Lett. 88, 034102 (2002). 
[20] K. Panajotov, M. Sciamanna, A. Tabaka, P. Mégret, M. Blondel, G. Giacomelli, F. Marin, H. Thienpont, and I. Veretennicoff, Phys. Rev. A 69, 011801(R) (2004).

[21] J. Houlihan, D. Goulding, Th. Busch, C. Masoller, and G. Huyet, Phys. Rev. Lett. 92, 050601 (2004).

[22] M. Arizaleta Arteaga, M. Valencia, M. Sciamanna, H. Thienpont, M. Lopez-Amo, and K. Panajotov, Phys. Rev. Lett. 99, 023903 (2007).

[23] G. Giacomelli, F. Marin, and I. Rabbiosi, Phys. Rev. Lett. 82, 675 (1999).

[24] S. Barbay, G. Giacomelli, and F. Marin, Phys. Rev. E 63, 051110 (2001).

[25] J. Danckaert et al., IEEE J. Sel. Top. Quantum Electron. 10, 911 (2004).

[26] D. W. Sukow, A. Gavrielides, T. Erneux, B. Mooneyham, K. Lee, J. McKay, and J. Davis, Phys. Rev. E 81, 025206(R) (2010).

[27] S. Jiang, Z. Pan, M. Dagenais, R. A. Morgan, and K. Kojima, Appl. Phys. Lett. 63, 3545 (1993).

[28] F. Robert, P. Besnard, M. L. Charès, G. Stéphan, IEEE J. Quantum Electron. QE-33, 2231 (1997).

[29] H. Li, A. Hohl, A. Gavrielides, H. Hou, and K. D. Choquette, Appl. Phys. Lett. 72, 2355 (1998).
[30] M. Sciamanna, T. Erneux, F. Rogister, O. Deparis, P. Mégret, and M. Blondel, Phys. Rev. A 65, 041801(R) (2002).

[31] M. Sciamanna, F. Rogister, O. Deparis, P. Mégret, M. Blondel, and T. Erneux, Opt. Lett. 27, 261 (2002).

[32] J. M. S. Solorio, D. W. Sukow, D. R. Hicks, and A. Gavrielides, Opt. Commun. 214, 327 (2002).

[33] T. Heil, A. Uchida, P. Davis, and T. Aida, Phys. Rev. A 68, 033811 (2003).

[34] A. Gavrielides, T. Erneux, D. W. Sukow, G. Burner, T. McLachlan, J. Miller, and J. Amonette, Opt. Lett. 31, 2006 (2006).

[35] A. Gavrielides, D. W. Sukow, G. Burner, T. McLachlan, J. Miller, and J. Amonette, Phys. Rev. E 81, 056209 (2010).

[36] Y. C. Kouomou, P. Colet, L. Larger, and N. Gastaud, Phys. Rev. Lett. 95, 203903 (2005).

[37] Y. Takahashi and H. Kawaguchi, IEEE J. Quantum Electron. 36, 864 (2000).

[38] J. Albert, G. Van der Sande, B. Nagler, K. Panajotov, I. Veretennicoff, J. Danckaert, and T. Erneux, Opt. Commun. 248, 527 (2005).

[39] C. J. Chang-Hasnain, J. P. Harbison, G. Hasnain, A. C. Von Lehmen, L. T. Florez, and N. G. Stoffel, IEEE J. Quantum Electron. 27, 1402 (1991). 